\title{
Application of artificial neural network for dimensional stability of repair materials under local hot weather
}

\author{
M. I. Khan \\ Department of Civil Engineering, King Saud University, Saudi Arabia
}

\begin{abstract}
In Saudi Arabia, the majority of concrete structures constructed more than three decades ago suffer because of lack of quality control and severe weather conditions. Concrete structures are prone to deterioration due to the very hot and harsh environmental conditions. In this paper, six candidate materials are reported. Mixture proportions, mixing and curing for all selected materials were employed as per the recommendations suggested by the manufacturer. Based on the experimentally obtained results, the applicability of an artificial neural network for the prediction of shrinkage has been established. The predicted values obtained using artificial neural networks have a good correlation between the experimentally obtained values. Therefore, it is possible to avoid shrinkage of repair materials using artificial neural networks.
\end{abstract}

Keywords: repair materials, dimensional stability, neural network, hot weather.

\section{Introduction}

In Saudi Arabia, the majority of concrete structures constructed more than three decades ago suffer because of lack of quality control and severe weather conditions. Concrete structures are prone to deterioration due to the very hot and harsh environmental conditions. Saudi Arabia covers a large area and is characterized by considerable differences in climate from one region to another, from the hot humid coastal areas to dry inland, and also by the extreme difference in temperature between winter and summer. Due to the hostile environment, the deterioration of concrete structures often takes place before their service life. Therefore, the structures deteriorate and need urgent repair. 
Shrinkage of repair material is the major factor inducing cracking in concrete repairs. The repair mortars are restrained against shrinkage by the substrate concrete and steel reinforcement within the repair patch. Induced cracks in repair materials are due to restrained shrinkage. The amount of shrinkage depends on many factors including the cement content, aggregate properties, the mixture composition, temperature and the relative humidity of the environment, the age of the concrete and the size of the structure.

Emmons et al. [1] reported that the shrinkage in the repair material can affect the durability of the repair patch along with the load carrying capacity of the repair patch. Stresses in a patch repair due to drying shrinkage for evaluation of concrete patching materials indicated that the shrinkage of the majority of the repair materials far exceeded the shrinkage strain value of a normal concrete of 500 microstrain at 30 days. The ASTM C 157 shrinkage test was used to determine the shrinkage values. Only $15 \%$ of the materials tested displayed a shrinkage which was less than that of its corresponding typical structural concrete tested in the same manner. Over 50\% of the materials had shrinkage strains twice as great as the plain concrete, despite the manufacturers' claims that they were expansive, non-shrinking or shrinkage compensated. In another study [2], the free shrinkage of a structural concrete tested was 450 microstrain at 90 days (storage conditions: $20^{\circ} \mathrm{C}$ and $55 \% \mathrm{RH}$ ). Only two of the thirteen commercially available repair materials (i.e. $15 \%$, similar to the ratio presented by Morgan [3] tested for shrinkage exhibited shrinkage strains lower than 450 microstrain, despite the manufacturers' claims that they were shrinkage compensated. $23 \%$ of the repair materials (as opposed to $50 \%$ presented by Morgan [3] had shrinkage strains twice as great as the structural concrete. In the recent research trend, the simulation modelling for repair materials are proposed [4] to predict its shrinkage more precisely. Property evaluation of magnesium phosphate cement mortar as patch repair material

Dector and Lambe [5] report on the Hong Kong Housing Authority (HKHA) specifications for repair mortars. Mortars are classified on the basis of their compressive strength, but other material properties which are equally important. The research conducted in the UK, $50 \%$ of the materials tested exhibited free shrinkage which was more than 300 microstrain at 7 days, but the larger specimen size of $100 \times 100 \times 500 \mathrm{~mm}$ (which gives a lower surface/volume ratio of 0.04 ) and storage conditions of $20^{\circ} \mathrm{C}$ and $55 \%$ RH differ to the HKHA (Hong Kong Housing Authority) test conditions.

The Australian standard for testing concrete, AS 1012, Part 3 also takes an interest in drying shrinkage [6]. A drying shrinkage of not greater than 450 microstrain at 28 days has been put forward. The UK Department of Transport's $1987 / 88$ maintenance programme included the use of shrinkage-compensated flowable concretes on two concurrent trial repair contracts on the Midland Links Motorway viaducts. Furthermore, in a recent publication, the volume stability measurements demonstrated that the drying shrinkage of magnesium phosphate cement (MPC) based repair mortars is much less than that of OPC mortar. It is demonstrated that MPC mortar is promising to be utilized in patch repair and maintenance works of concrete [7]. 
Shrinkage of a repair material can be obtained by preparing samples and monitoring the deformation which occurs over a period of time. Shrinkage is very much affected by the specimen size and shape and storage conditions [2, 4, 8].

This indicated that repair products are not being designed to minimize shrinkage, despite the claim that the materials available commercially are nonexpansive, non-shrinking or shrinkage compensating. Moreover, it was found that only limited information on the properties was available from manufacturers' data sheets. Information regarding shrinkage was not even listed on some of them. It has been reported that the industry cannot limit the manufacturers of repair materials to a certain maximum shrinkage value because the basis for an acceptable shrinkage value has not yet been established.

\section{Materials and testing procedure}

\subsection{Materials}

Seven candidate materials, three from each cementitious repair mortars and cementitious polymer modified mortars, were selected. The selection of the repair material (RM) was based on the popularity and best-selling materials manufactured by the top three companies Fosroc, Sika and MBT available in the Kingdom of Saudi Arabia. The designation, properties and application of these cementitious repair mortars (CRM) and cementitious polymer modified mortars (CPMM) are shown in Table 1. Mixture proportions, mixing and curing for all selected materials were employed as per the recommendations suggested by the manufacturer.

\subsection{Shrinkage measurement}

To measure the free drying shrinkage of concrete, $76 \times 76$ x $285 \mathrm{~mm}$ prism's were cast in accordance with ASTM C 157-94b. For mortar, 25 x 25 x 285 mm prism's were cast and tested in accordance with ASTM 1012-89. The drying shrinkage values were determined for each material under two different environments (20 and 50 percent relative humidity) and the specimens were monitored up to a period of 12 months. In addition, some specimens were also exposed to the actual field conditions.

\subsection{Environmental condition}

Drying shrinkage was monitored for all types of repair materials under investigation in this project. For this test three environmental conditions were employed as follows:

Air Dry (Lab Environment): $\quad$ Temperature $23 \pm 2^{0} \mathrm{C}$; Humidity $25 \pm 5 \%$

Humid (Humidity Room): Temperature $28 \pm 2^{0} \mathrm{C}$; Humidity $65 \pm 5 \%$

Outside:

Open Environment of Riyadh 
These environmental conditions were used taking into account the local environmental conditions into consideration. Samples were also kept outside the laboratory in the open environment conditions of Riyadh Region between January and March. These samples were outside for almost one year and encountered all weather conditions of Riyadh.

Table 1: $\quad$ Properties of repair materials used in the investigation.

\begin{tabular}{|c|c|c|c|}
\hline Material & Designation & Properties & Application \\
\hline \multirow{4}{*}{$\begin{array}{l}\text { Cementitious } \\
\text { repair mortars }\end{array}$} & CRM-1 & $\begin{array}{l}\text { Shrinkage } \\
\text { controlled }\end{array}$ & $\begin{array}{l}\text { Hand applied, } \\
\text { Vertical and Overhead }\end{array}$ \\
\hline & CRM-2 & $\begin{array}{l}\text { Shrinkage } \\
\text { controlled }\end{array}$ & $\begin{array}{l}\text { Spraying, } \\
\text { Vertical and Overhead }\end{array}$ \\
\hline & CRM-3 & $\begin{array}{l}\text { Multi purpose, } \\
\text { For hot climates }\end{array}$ & $\begin{array}{l}\text { Hand applied, } \\
\text { Vertical and Horizontal }\end{array}$ \\
\hline & CRM-4 & $\begin{array}{l}\text { Multi purpose, } \\
\text { For hot climates }\end{array}$ & $\begin{array}{l}\text { Spraying, } \\
\text { Vertical and Horizontal }\end{array}$ \\
\hline \multirow{3}{*}{$\begin{array}{l}\text { Cementitious } \\
\text { polymer modified } \\
\text { mortars }\end{array}$} & CPMM-1 & $\begin{array}{l}\text { Multi purpose, } \\
\text { For hot climates }\end{array}$ & $\begin{array}{l}\text { Dry/Wet spray } \\
\text { Vertical, Overhead and } \\
\text { Horizontal }\end{array}$ \\
\hline & СРMM-2 & For hot climates & $\begin{array}{l}\text { Wet spray, } \\
\text { Vertical and Overhead }\end{array}$ \\
\hline & CPMM-3 & Multi purpose & Hand applied \\
\hline
\end{tabular}

\section{Results and discussion}

\subsection{Cementitious repair mortar}

\subsubsection{Hand applied}

Drying shrinkage of CRM-2 and Sika Rep is presented in Figures 1 and 2, respectively. Both CRM-2 and Sika Rep showed expansion at the initial stage, this is may be because the specimens were cured in water for 28 days in accordance with ASTM specifications. After 28 days all specimens were placed in their respective environments to monitor shrinkage. After about 28 days, there is a sharp increase in shrinkage up until about 100 days and then a steady increase is maintained.

Drying shrinkage of CRM-2 cured in the Lab environment and cured outside increased continuously up until about 150 days as can be seen from the steep curves as shown in Figure 1. At 150 days the shrinkage of CRM-2 is about 1700 microns and after 150 days the drying shrinkage remained almost constant, as can be seen from the flat curves (Figure 1). CRM-2 cured in humid room showed lower shrinkage throughout. 


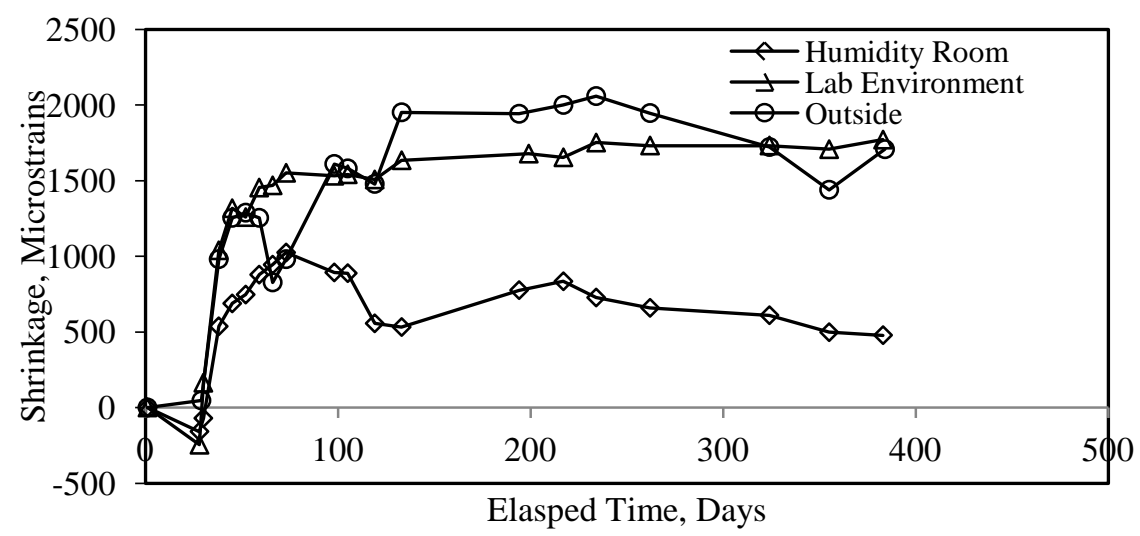

Figure 1: $\quad$ Drying shrinkage of CRM-2 (Hand applied).

The pattern of drying shrinkage of CRM-3 cured in the Lab environment and cured outside is similar to that of CRM-2. However, the maximum shrinkage was restricted to about 1400 microns at about 200 days Figure 2. Similarly, humid cured CRM-3 shrinkage was in also of the same pattern as CRM-2. The comparison of 28-day shrinkage of CRM-2 and CRM-3 is presented in Figure 3. From this figure it can be seen that at 28-days, CRM-3 showed expansion for all curing environments and CRM-2 showed expansion for all environments except the Riyadh Outside environment.

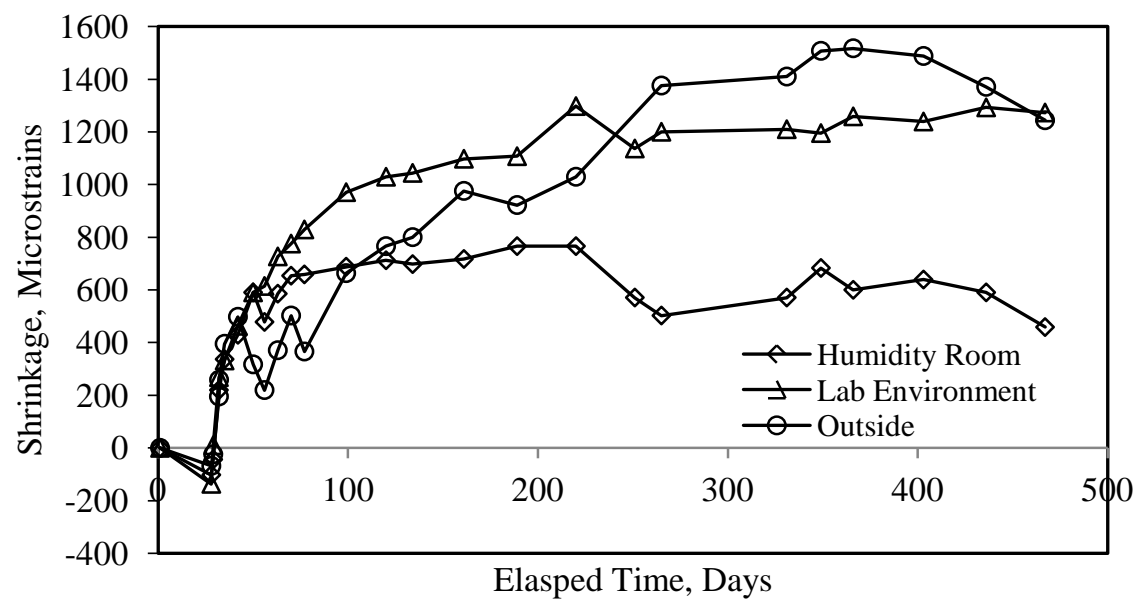

Figure 2: $\quad$ Drying shrinkage of CRM-3 (Hand applied). 


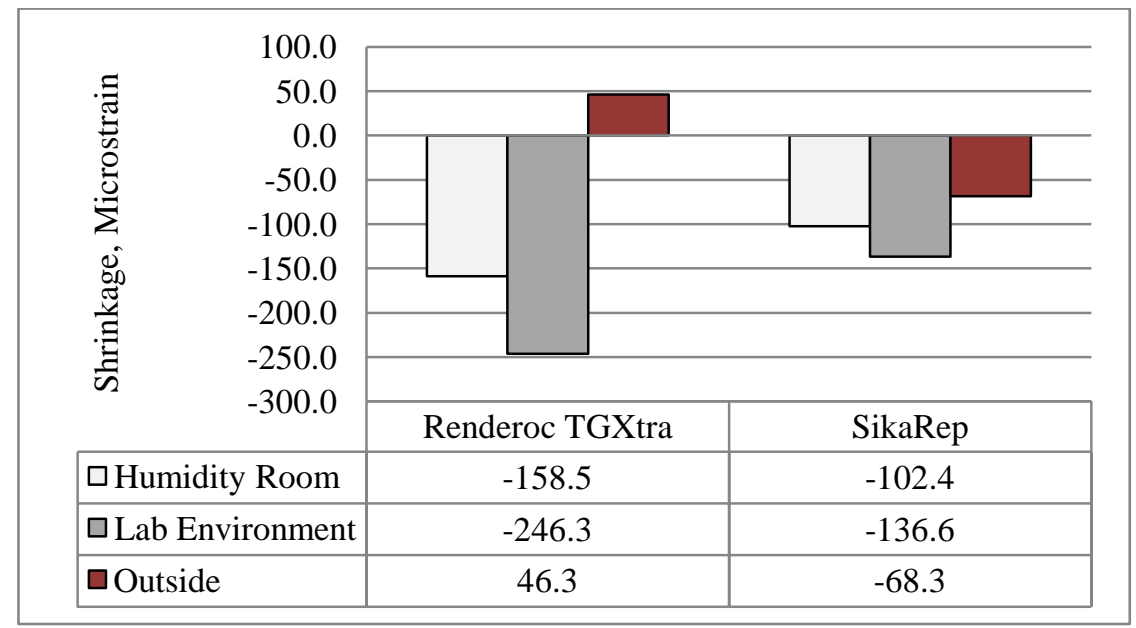

Figure 3: $\quad$ Drying shrinkage of cementitious repair mortars at 28 days.

\subsubsection{Spray applied}

Drying shrinkage of CRM-1 and CRM-4 is presented in Figures 4 and 5, respectively. Both CRM-1 and CRM-4 showed expansion at an initial stage. After 28 days, all specimens were in their respective environments to monitor shrinkage. After about 28 days, there is a sharp increase in shrinkage for up to 100 days and then a steady increase is maintained.

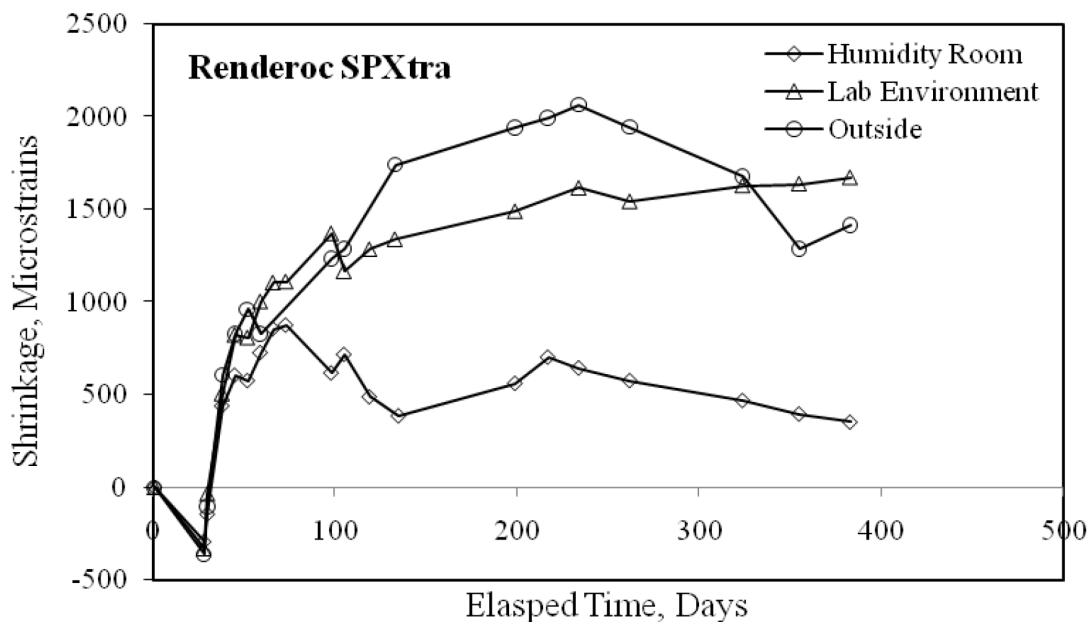

Figure 4: $\quad$ Drying shrinkage of CRM-1 (Spray applied). 


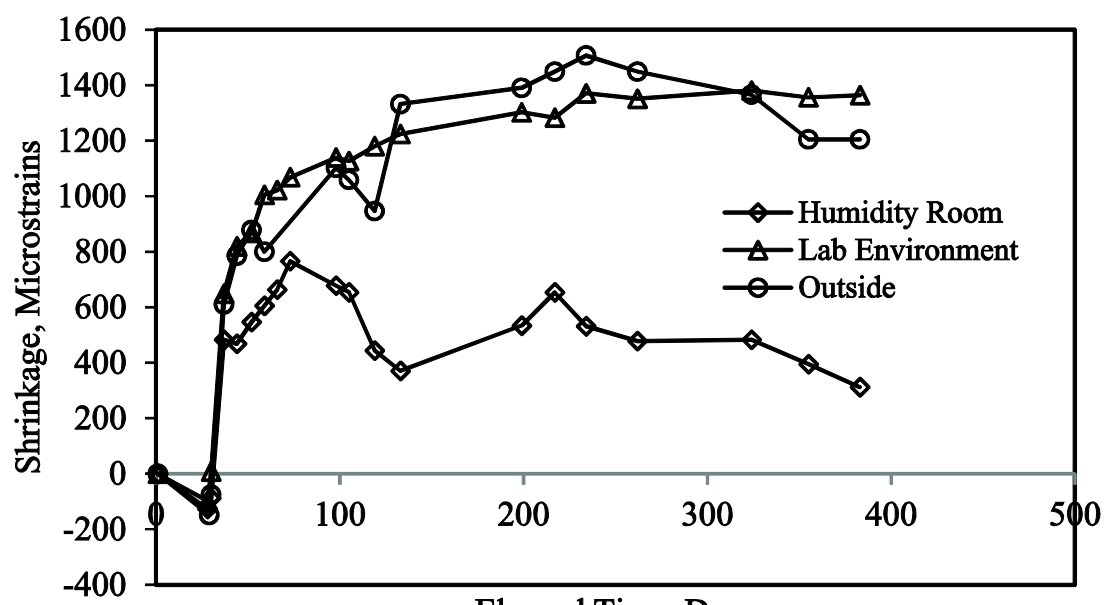

Elasped Time, Days

Figure 5: $\quad$ Drying shrinkage of CRM-4 (Spray applied).

Drying shrinkage of CRM-1 cured in the Lab environment and cured outside increased continuously up until about 150 days and reaches 2000 microns and 1500 microns for the outside and lab environments, respectively. After 150 days the shrinkage remained almost constant. CRM-1 cured in the humid room showed a lower shrinkage value of 800 microns at around 90 days (Figure 4).

The drying shrinkage of CRM-4 cured in the Lab environment and cured outside increases sharply up until about 100 days then there is steady increase up to about 250 days during which the shrinkage value reaches 1400 microns (Figure 5). Humid cured CRM-4 showed almost similar results and pattern as that of CRM-1. The comparison of 28-day shrinkage of CRM-1 and CRM-4 is presented in Figure 6. From this figure it can be seen that at 28-days both CRM1 and CRM-4 showed expansion for all curing conditions.

\subsection{Cementitious polymer modified mortar}

Drying shrinkage of CPMM-1 is presented in Figure 7. From the figure, it can be seen that all curing regimes showed expansion at an initial stage, for the reasons discussed in previous sections. After about 28 days, there is a sharp increase in shrinkage up to about 50 days and then a steady increase is maintained.

The drying shrinkage for lab cured CPMM-1 specimen is about 1400 microns at 70 days then there is a steady increase until it reaches 1500 microns at about 250 days and after that the shrinkage remains constant. Outside cured specimen has a sharp increase until 70 days and then a steady increase up to 2000 microns at 250 days, whereas shrinkage for humid cured sample reaches about 600 microns at 60 days and then remains constant. 


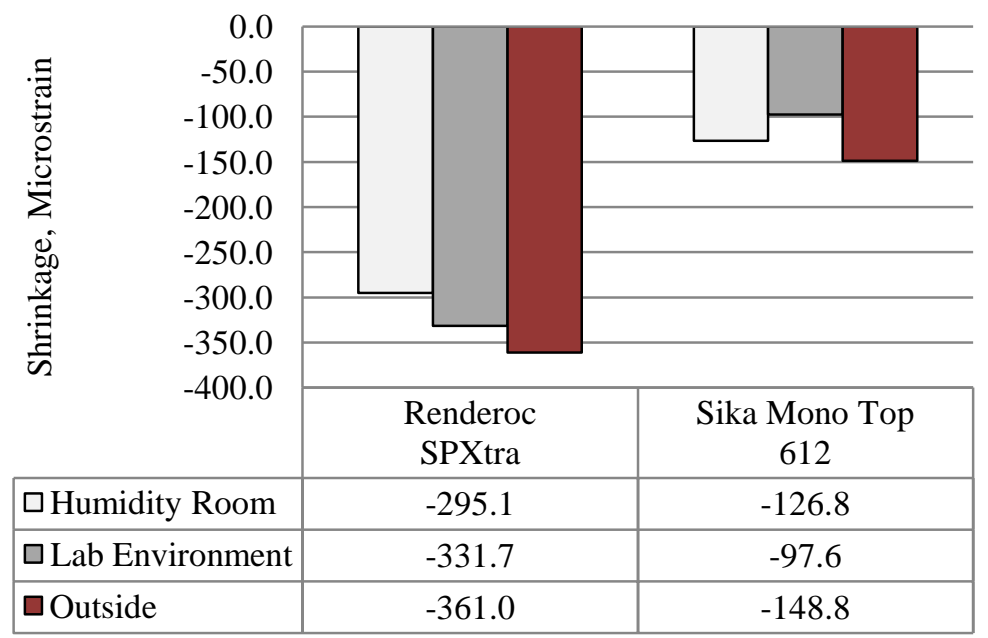

Figure 6: $\quad$ Drying shrinkage of cementitious repair mortars at 28 days.

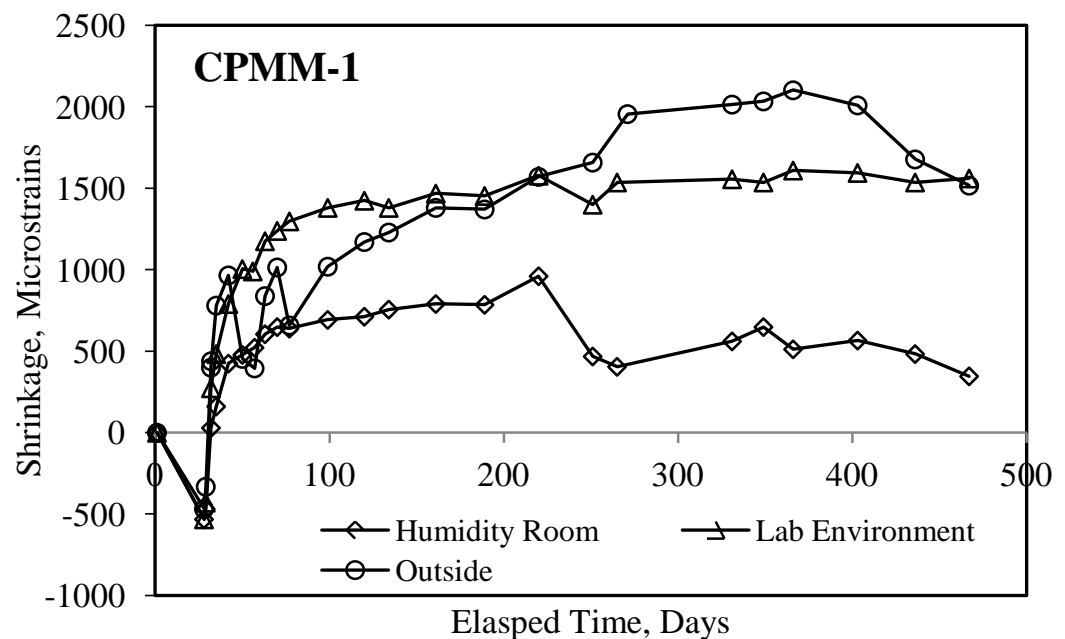

Figure 7: $\quad$ Drying shrinkage of CPMM-1.

The comparison of 28-day shrinkage of CPMM-1 and CPMM-2 is presented in Figure 8. From this figure it is clear that at 28-days CPMM-1 and CPMM-2 showed expansion for all curing conditions. However, expansion of CPMM-1 is almost three times higher than that of CPMM-2. 


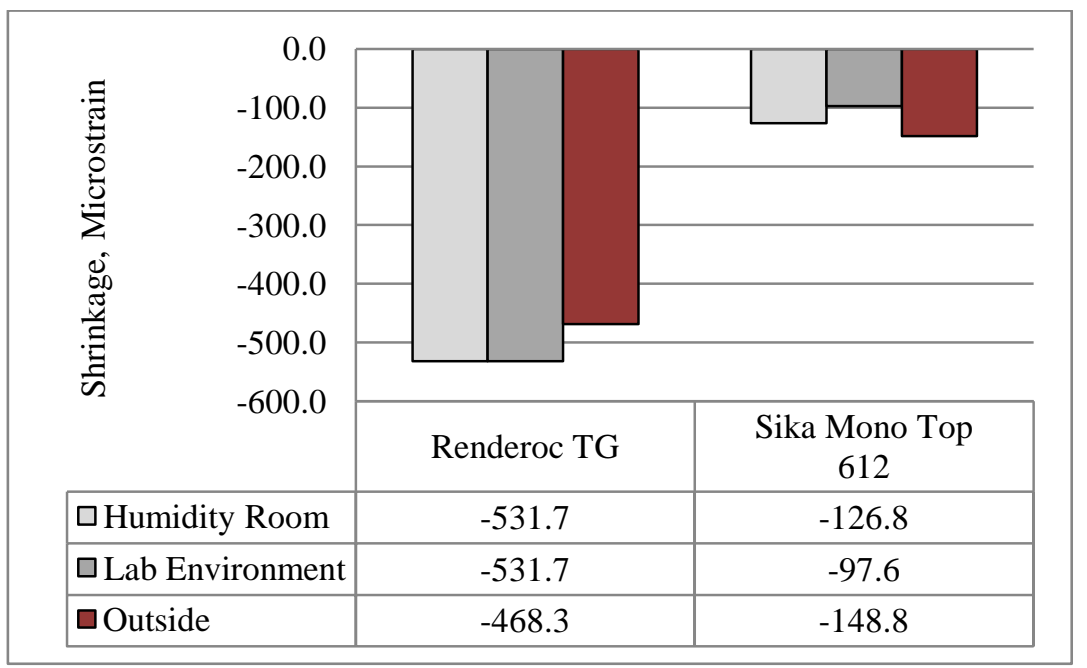

Figure 8: Drying shrinkage of cementitious polymer modified repair mortars.

\section{Artificial neural networks}

A radial basis function (RBF) network can be regarded as a special two layer network which is linear in the parameters provided all the RBF centres are prefixed. Given fixed centres i.e. no adjustable parameters the first layer or the hidden layer performs a fixed nonlinear transformation, which maps the input space onto a new space. The output layer then implements a linear combiner on this new space and the only adjustable parameters are the weights of this linear combiner. These parameters can therefore be determined using the linear least square method, which is an important advantage of this approach. A schematic of the RBF network with $n$ inputs and a scalar output is shown in Figure 9 and is represented as

$$
\hat{y}(t)=w_{0}+\sum_{i=1}^{n} w_{i} f\left(\left\|x(t)-c_{i}\right\|\right)
$$

where $\hat{y}(t)$ is the network predicted output; $x(t)$ is the network's input vector and presented as follows:

$$
x(t)=\left[\left(y(t-1), \ldots y\left(t-n_{y}\right), u(t-1), \ldots u\left(t-n_{u}\right)\right]^{T}\right.
$$

$w_{i}$ are the weights or parameters; $c_{i}$ are known as RBF centres. Once the functional form $f($.$) and the centres c_{i}$ are fixed, and the set of input $x(t)$ and the corresponding desired output vector $\left(y(t)\right.$ in this study) provided, the weights $w_{i}$ can be determined using the linear least squares method. Assuming the RBF network in Eq. (1) as a special case of the linear regression model is presented as follows:

$$
y(t)=\sum_{i=1}^{M} p_{i}(t) \theta_{i}+\varepsilon(t)
$$


where $y(t)$ is the desired output; $p_{i}$ are known regressors, which are some nonlinear functions of lagged outputs and inputs, as follows:

$$
p_{i}(t)=p_{i}(x(t))
$$

A constant term ( $w_{0}$ in Figure 9$)$ can be included in Eq. (3) by setting the corresponding term $p_{i}(t)=1$. The residual $\varepsilon(t)$ is assumed to be uncorrelated with the regressors $p_{i}(t)$. It is clear that a given centre $c_{i}$ with a given nonlinear function $f($.$) corresponds to p_{i}(t)$ in Eq. (4). Eq. (4) for $t=1, \ldots N$, can be written in the matrix form

$$
y=P \Theta+E
$$

The network developed in this investigation has input layer and two units in the output layer. The experimentally obtained data have been divided into two sets, one for the network learning called learning set, and the other for testing the network called testing set. Each set is composed of dozens of pairs of input vectors and output vectors (vectors in the input layer called input vectors, and in the output layer called output vectors). An input vector consists of ingredient components which influence the output vector, shrinkage. The predicted values obtained using ANN were plotted against their respective experimentally obtained values as shown in Figure 10. It can be seen that there is a good correlation between experimental values and those predicted using ANN. Based on the experimentally obtained results, ANN has been used to establish its applicability for the prediction purposes. Mix proportion can be predicted using can be predicted using ANN. However, some trail mixes are necessary for better performance and elimination of material variability from place to place. There is a good correlation between experimental values and those predicted using ANN. Based on the experimentally obtained results, ANN has been used to establish its applicability for the prediction purposes. Shrinkage can be predicted using can be predicted using ANN. However, some trail mixes are necessary for better performance and elimination of material variability.

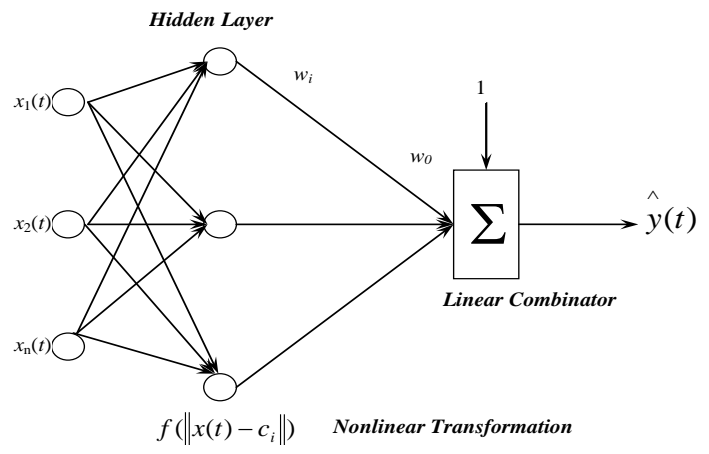

Figure 9: $\quad$ Radial basis function network. 


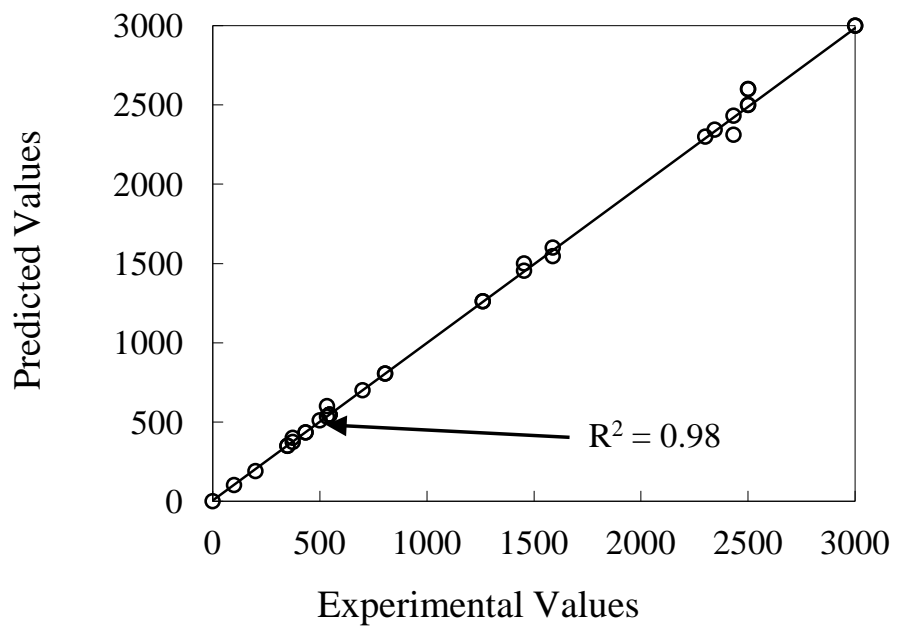

Figure 10: Relationship between experimental and predicted values of shrinkage using ANN.

\section{Conclusions}

- Almost all the materials cured in the lab environment and outside showed higher drying shrinkage as compared to the humid cured specimens.

- CPMM-1, CRM-1 and CRM-2 cured in the lab and outside environments showed drying shrinkage as high as about 2000 microns whereas the shrinkage in humid cured material specimens was limited to about $700-800$ microns.

- CPMM-2 and CRM-3 cured in the lab environment and outside showed drying shrinkage as high as about 1400 microns.

- Based on the experimentally obtained results, ANN has been used to establish its applicability for the prediction purposes. Shrinkage of RM can be predicted using can be predicted using ANN. However, some trail mixes are necessary for better performance and elimination of material variability.

\section{References}

[1] Emmons, P.H., Vaysburd, A.M., McDonald, J.E., Concrete repair in the future turn of the century - Any problems? Concrete International, pp. 4249, 1994.

[2] Mangat, P.S., Limbachiya, M.K., Repair material properties for effective structural application. Cement and Concrete Research, Vol. 27, No. 4, pp. 601-617, 1997. 
[3] Morgan, D.R., Compatibility of concrete repair materials and systems. Construction and Building Materials, Vol 10, No.1, pp. 57-67, 1996.

[4] Abbasnia, R., Godossi P. and Ahmadi J., Prediction of restrained shrinkage based on restraint factors in patching repair mortar. Cement and Concrete Research, Vol. 35, pp. 1909-1913, 2005.

[5] Dector, M.H.; Lambe, R.W., New materials for concrete repair Development and testing. The Indian Concrete Journal, pp. 475-480, October 1993.

[6] Australian Standards, Methods of testing concrete - methods for the determination of properties related to the consistence of concrete. AS 1012, Part 3, 1992.

[7] Qiao, F., Chau, C.K. and Li Z., Property evaluation of magnesium phosphate cement mortar as patch repair material. Construction and Building Materials, Vol. 24, pp. 695-700, 2010.

[8] Coutinho, A., The influence of the type of cement on its cracking tendency. RILEM Bulletin, 5, pp. 26-40, December 1959. 